\title{
AVERAGES OF SIMPLEX HILBERT TRANSFORMS
}

\author{
POLONA DURCIK AND JORIS ROOS
}

(Communicated by Alexander Iosevich)

\begin{abstract}
We study a multilinear singular integral obtained by taking averages of simplex Hilbert transforms. This multilinear form is also closely related to Calderón commutators and the twisted paraproduct. We prove $L^{p}$ bounds in dimensions two and three and give a conditional result valid in all dimensions.
\end{abstract}

\section{INTRODUCTION} by

$$
\int_{\mathbb{R}^{n}} p . v \cdot \int_{\mathbb{R}} \int_{[0,1]^{n-1}} F_{0}(x)\left(\prod_{j=1}^{n-1} F_{j}\left(x+t \alpha_{j} e_{j}\right)\right) F_{n}\left(x+t e_{n}\right) d \alpha \frac{d t}{t} d x,
$$

where $e_{1}, \ldots, e_{n}$ are the standard unit vectors in $\mathbb{R}^{n}$. We prove the following result.

Theorem 1. Let $n$ equal two or three. Let $p_{0} \in\left(2^{n-1}, \infty\right), p_{1}, \ldots, p_{n} \in(1, \infty)$, and $\sum_{j=0}^{n} p_{j}^{-1}=1$. If $n=3$, assume in addition that $\max \left(p_{1}, p_{2}, p_{3}\right)<4$. Then there exists a constant $c \in(0, \infty)$ such that

$$
\left|\Lambda^{(n)}\left(F_{0}, \ldots, F_{n}\right)\right| \leq c \prod_{j=0}^{n}\left\|F_{j}\right\|_{p_{j}} .
$$

The constant $c$ depends on $p_{0}, p_{1}, \ldots, p_{n}$. The estimate fails if $p_{j}=\infty$ for some $j=1, \ldots, n$.

If we drop the average over $\alpha$ in (1.1) and fix, say $\alpha=(1, \ldots, 1)$, then we obtain the simplex Hilbert transform

$$
\int_{\mathbb{R}^{n}} p . v \cdot \int_{\mathbb{R}} F_{0}(x)\left(\prod_{j=1}^{n-1} F_{j}\left(x+t e_{j}\right)\right) F_{n}\left(x+t e_{n}\right) \frac{d t}{t} d x .
$$

Note that for $n=1$, both (1.1) and (1.3) reduce to a dualized form of the classical Hilbert transform. For $n \geq 2$, it is currently not known whether the multilinear form (1.3) satisfies any $L^{p}$ estimates. This is a major open problem in harmonic analysis. In the case $n=2$, the form (1.3) is also called a triangular Hilbert transform and some partial progress has been made in [KTZ15], where bounds for a Walsh model are obtained, when one of the three functions takes a special form.

Received by the editors December 31, 2018.

2010 Mathematics Subject Classification. Primary 42B20; Secondary 42B15.

Key words and phrases. Multilinear singular integrals, simplex Hilbert transform, paraproducts. 
$L^{p}$ bounds for the triangular Hilbert transform would unify several known results in time-frequency analysis, such as uniform bounds for the bilinear Hilbert transform and bounds for Carleson's operator, which controls pointwise almost-everywhere convergence of Fourier series. More generally, bounds for the $n$-simplex Hilbert transform would imply bounds for the $n$-linear Hilbert transform

$$
\int_{\mathbb{R}} p \cdot v \cdot \int_{\mathbb{R}} f_{0}(x) f_{1}(x+t) f_{2}(x+2 t) \cdots f_{n}(x+n t) \frac{d t}{t} d x .
$$

We refer to [KTZ15, Appendix B] and [Zor17, Appendix A] for the details of this implication. If $n=2$, then the previous display represents a trilinear form dual to the bilinear Hilbert transform, which was first shown to satisfy $L^{p}$ bounds by Lacey and Thiele [LT97], [LT99]. If $n \geq 3$, then it is not known if (1.4) satisfies any $L^{p}$ bounds and this is considered to be a difficult open problem. An averaged form of the 3-linear Hilbert transform was studied by Palsson Pal12. Tao [Tao16] showed that truncations of the multilinear form (1.4) exhibit some non-trivial cancellation by using regularity lemmas from arithmetic combinatorics. For the more general simplex Hilbert transform (1.3), a similar result was obtained by Zorin-Kranich [Zor17] and this was strengthened subsequently in DKT16].

The multilinear form (1.1) is closely related to the forms dual to Calderón commutators, which can be written as

$$
\int_{\mathbb{R}} p \cdot v \cdot \int_{\mathbb{R}} \int_{[0,1]^{n-1}} f_{0}(x)\left(\prod_{j=1}^{n-1} f_{j}\left(x+t \alpha_{j}\right)\right) f_{n}(x+t) d \alpha \frac{d t}{t} d x
$$

for a suitable choice of the functions $f_{j}$. In fact, $L^{p}$ bounds for (1.1) imply the same $L^{p}$ bounds for (1.5). This follows in the same way as bounds for the simplex Hilbert transform would imply bounds for (1.4). Calderón commutators played a key role in the resolution of the problem of bounding the Cauchy integral along Lipschitz curves by Coifman, McIntosh, and Meyer CMM82. More recently, Muscalu [Mus14a, Mus14b] developed an alternative approach for bounding Calderón commutators, which also inspired our proof of Theorem 1. We remark that in view of the connection with the Cauchy integral, the focus for bounding commutators often lies in obtaining $L^{\infty}$ bounds and a constant that depends polynomially on $n$. In sharp contrast to this, the estimate (1.2) fails if $p_{j}=\infty$ for some $j \in\{1, \ldots, n\}$. We prove this in 8

Another object that is intimately related to (1.1) is the twisted paraproduct

$$
\int_{\mathbb{R}^{4}} F_{0}(x, y) F_{1}(x-t, y) F_{2}(x, y-s) K(t, s) d(t, s, x, y),
$$

where $K$ is a Calderón-Zygmund kernel on $\mathbb{R}^{2}$. This operator first arose as a degenerate case of the two-dimensional bilinear Hilbert transform that did not fall within the scope of Demeter and Thiele's time-frequency techniques [DT10]. $L^{p}$ bounds were obtained by Kovač Kov12, who used a novel argument involving repeated use of the Cauchy-Schwarz inequality and a telescoping identity. These techniques have since been further developed and applied to other problems (including the application to the simplex Hilbert transform [DKT16] which we have already mentioned). 
Let $m$ be a bounded function on $\mathbb{R}^{n} \backslash\{0\}$. Then we define

$$
\Lambda_{m}\left(F_{0}, \ldots, F_{n}\right)=\int_{\left(\mathbb{R}^{n}\right)^{n}} \widehat{F_{0}}\left(-\left(\tau_{1}+\cdots+\tau_{n}\right)\right)\left(\prod_{j=1}^{n} \widehat{F_{j}}\left(\tau_{j}\right)\right) m\left(\tau_{11}, \ldots, \tau_{n n}\right) d \tau
$$

where $\tau=\left(\tau_{1}, \ldots, \tau_{n}\right) \in\left(\mathbb{R}^{n}\right)^{n}$ and $\tau_{j}=\left(\tau_{j 1}, \ldots, \tau_{j n}\right) \in \mathbb{R}^{n}$. The key property here is that the multiplier only depends on the diagonal frequency variables $\tau_{11}, \ldots, \tau_{n n}$. The form (1.6) is obtained by specializing $n=2$. If we set

$$
\mu_{n}(\xi)=\int_{[0,1]^{n-1}} \operatorname{sgn}\left(\alpha_{1} \xi_{1}+\cdots+\alpha_{n-1} \xi_{n-1}+\xi_{n}\right) d \alpha,
$$

then $\Lambda_{\mu_{n}}$ coincides with our multilinear form (1.1) (up to a multiplicative constant). The symbol (1.8) also coincides with the symbol of the Calderón commutator (1.5). The function $\mu_{n}$ is continuous on $\mathbb{R}^{n} \backslash\{0\}$ but fails to be differentiable on several hyperplanes. Some details and further comments on the function $\mu_{n}$ are contained in $\$ 5$

We call a bounded function $m \in C^{\infty}\left(\mathbb{R}^{n} \backslash\{0\}\right)$ a standard smooth symbol if for every $\alpha \in \mathbb{N}_{0}^{n}$ there exists $c_{\alpha} \in(0, \infty)$ such that for every $\xi \in \mathbb{R}^{n} \backslash\{0\}$ we have

$$
\left|\partial^{\alpha} m(\xi)\right| \leq c_{\alpha}|\xi|^{-|\alpha|} .
$$

Notice that $\mu_{n}$ is not a standard smooth symbol. Moreover, note that even if $m$ is a standard smooth symbol, the multilinear Fourier multipliers of the form (1.7) do not belong to the multilinear Calderón-Zygmund paradigm as set forth in [GT02].

Our proof of Theorem 11 relies on the estimate

$$
\left|\Lambda_{m}\left(F_{0}, \ldots, F_{n}\right)\right| \leq c \prod_{j=0}^{n}\left\|F_{j}\right\|_{p_{j}}
$$

for standard smooth symbols $m$ with the constant $c$ only depending on $m$. In fact, we prove the following conditional result.

Theorem 2. Let $n \geq 2$. If the estimate (1.10) holds for all standard smooth symbols $m$ and some fixed exponents $p_{0}, \ldots, p_{n} \in(1, \infty)$, then (1.2) holds (for the same exponents).

In the case $n=2$, Kovač Kov12] showed that (1.10) holds for all $p_{0} \in(2, \infty), p_{1}$, $p_{2} \in(1, \infty)$ satisfying $p_{0}^{-1}+p_{1}^{-1}+p_{2}^{-1}=1$.

In the case $n=3$ we are still able to use the techniques from Kov12 to prove (1.10) for all $p_{0} \in(4, \infty), p_{1}, p_{2}, p_{3} \in(1,4)$ satisfying $p_{0}^{-1}+p_{1}^{-1}+p_{2}^{-1}+p_{3}^{-1}=1$. Indeed, the proof is by reduction to a result for a certain dyadic form considered in Kov12, combined with a transition from the dyadic to the continuous case and fiber-wise Calderón-Zygmund decomposition by Bernicot [Ber12]. These arguments are well known, but for the convenience of the reader some details are given in $\$ 3$.

We currently do not have the estimate (1.10) for any $n \geq 4$ (for any tuple of exponents $\left.\left(p_{j}\right)_{j}\right)$. This problem is closely related to the question of extending the range of exponents for the twisted paraproduct (1.6), as well as proving $L^{p}$ estimates for the integrals studied in DT18, in the case when the index set is an arbitrary subset of the cube in $\mathbb{R}^{n}$. However, in $\oiint 2$ we show that (1.2) holds provided that (1.10) holds. 
Organization of the paper. In $\sqrt{2}$ we prove Theorem 2 , In $\sqrt{3}$ we prove the estimate (1.10) for $n=3$. In $\$ 4$ we give a counterexample to (1.2) if $p_{j}=\infty$ for some $j \in\{1, \ldots, n\}$. In $\$ 5$ we make some comments about the symbol $\mu_{n}$.

\section{Main ARgument}

In this section we fix exponents $p_{0}, \ldots, p_{n} \in(1, \infty)$ and prove that (1.2) holds under the assumption that for all standard smooth symbols $m$ we have

$$
\left|\Lambda_{m}\left(F_{0}, \ldots, F_{n}\right)\right| \lesssim \prod_{j=0}^{n}\left\|F_{j}\right\|_{p_{j}}
$$

where $\Lambda_{m}$ is defined in (1.7).

First we make some comments about terminology. We say that a function $\psi$ of one real variable is of $\psi$-type if it is a smooth function compactly supported in an interval not containing the origin. The letter $\psi$ will always denote a $\psi$-type function supported on $\left[-2,-\frac{1}{2}\right] \cup\left[\frac{1}{2}, 2\right]$ such that $\sum_{k \in \mathbb{Z}} \psi\left(2^{-k} \eta\right)=1$ for all $\eta \neq 0$. We also reserve the letters $\widetilde{\psi}, \psi^{1}, \psi^{2}, \ldots$ for other $\psi$-type functions, which may change throughout the text.

Similarly, a function $\varphi$ of one real variable is of $\varphi$-type if it is a smooth function that is compactly supported. The letters $\varphi, \widetilde{\varphi}, \varphi^{1}, \varphi^{2}, \ldots$ are reserved for $\varphi$-type functions, which may change at different stages of the argument.

If $\varphi$ is a function and $k \in \mathbb{Z}$ an integer, then we write $\varphi_{k}(\eta)=\varphi\left(2^{-k} \eta\right)$.

We start by decomposing $\mathbb{R}^{n}$ into a family of Whitney boxes. Then we have

$$
1=\sum_{k_{1}, \ldots, k_{n} \in \mathbb{Z}} \psi_{k_{1}}\left(\xi_{1}\right) \cdots \psi_{k_{n}}\left(\xi_{n}\right)
$$

for almost every $\xi \in \mathbb{R}^{n}$. We split the sum over $\left(k_{1}, \ldots, k_{n}\right) \in \mathbb{Z}^{n}$ according to which of the components $k_{j}$ is the largest:

$$
1=\sum_{k_{1} \geq k_{2}, \ldots, k_{n}}(\cdots)+\sum_{k_{2} \geq k_{1}, k_{3}, \ldots, k_{n}, k_{2} \neq k_{1}}(\cdots)+\cdots+\sum_{k_{n}>k_{1}, \ldots, k_{n-1}}(\cdots) .
$$

For each of the $n$ terms in the previous display, we perform a further decomposition. Here we have that $k_{1}$ is no smaller than $k_{2}, \ldots, k_{n}$. We want to distinguish the case that $k_{1}$ is much larger than all of the remaining integers $k_{2}, \ldots, k_{n}$ from the case that $k_{1}$ is roughly equal to at least one of the numbers $k_{2}, \ldots, k_{n}$. To be more precise, we fix a large integer $\ell_{0} \gg 1$. For example, $\ell_{0}=100 \log (n)$ will do. Then decompose the corresponding summation as

$$
\sum_{k_{1} \geq k_{2}, \ldots, k_{n}}(\cdots)=\sum_{k_{1}-\ell_{0} \geq k_{2}, \ldots, k_{n}}(\cdots)+\sum_{\substack{k_{1} \geq k_{2}, \ldots, k_{n}, \exists j \in\{2, \ldots, n\} \text { s.t. } k_{1}-\ell_{0}<k_{j}}}(\cdots) .
$$

We say that the first of these two terms is of $\psi$ - $\varphi$-type. This is because we can sum in $k_{2}, \ldots, k_{n}$ to obtain

$$
\begin{gathered}
\sum_{k_{1}-\ell_{0} \geq k_{2}, \ldots, k_{n}} \psi_{k_{1}}\left(\xi_{1}\right) \cdots \psi_{k_{n}}\left(\xi_{n}\right)=\sum_{k_{1} \in \mathbb{Z}} \psi_{k_{1}}\left(\xi_{1}\right) \prod_{j=2}^{n} \sum_{k_{j} \leq k_{1}+\ell_{0}} \psi_{k_{j}}\left(\xi_{j}\right) \\
=\sum_{k \in \mathbb{Z}} \psi_{k}\left(\xi_{1}\right) \varphi_{k}\left(\xi_{2}\right) \cdots \varphi_{k}\left(\xi_{n}\right) .
\end{gathered}
$$


Similarly, the second term in (2.3) can be written as a sum over $O(n)$ terms of the form

$$
\sum_{k \in \mathbb{Z}} \psi_{k}^{1}\left(\xi_{1}\right) \psi_{k}^{j_{1}}\left(\xi_{j_{1}}\right) \prod_{j \neq j_{1}} \varphi_{k}^{j}\left(\xi_{j}\right),
$$

where $\psi^{j}, \varphi^{j}$ are $\psi$ - and $\varphi$-type functions, respectively. We refer to such a term as being of $\psi$ - $\psi$-type.

Summarizing, we obtain a partition of unity into $O\left(n^{2}\right)$ terms each of which is either of $\psi$ - $\varphi$-type or of $\psi$ - $\psi$-type. Now we write our multilinear form as

$$
\int_{\left(\mathbb{R}^{n}\right)^{n}} \widehat{F_{0}}\left(-\left(\tau_{1}+\cdots+\tau_{n}\right)\right) \widehat{F_{1}}\left(\tau_{1}\right) \cdots \widehat{F_{n}}\left(\tau_{n}\right) \mu_{n}\left(\tau_{11}, \ldots, \tau_{n n}\right) d\left(\tau_{1}, \ldots, \tau_{n}\right),
$$

and split the symbol $\mu_{n}$ into $O\left(n^{2}\right)$ pieces according to the aforementioned partition of unity. At this point our argument splits into three cases.

Case 1 (The $\psi$ - $\psi$ case). Symbols of $\psi$ - $\psi$-type can be handled using an argument similar to Muscalu's alternative proof [Mus14b] of the Coifman-McIntosh-Meyer theorem. This is detailed in $\$ 2.1$

Case 2 (The smooth $\psi-\varphi$ case). For the $\psi$ - $\varphi$-type pieces we distinguish two separate cases. The first scenario is that the $\psi$ falls onto the $n$th frequency variable (corresponding to the last term in (2.2) $)$. Here we are concerned with the symbol

$$
\sum_{k \in \mathbb{Z}} \mu_{n}(\xi) \varphi_{k}\left(\xi_{1}\right) \cdots \varphi_{k}\left(\xi_{n-1}\right) \psi_{k}\left(\xi_{n}\right)
$$

where $\varphi$ is supported in $\left[-2^{-\ell_{0}+1}, 2^{-\ell_{0}+1}\right]$. Recalling that $\psi$ is supported in $\left[-2,-\frac{1}{2}\right]$ $\cup\left[\frac{1}{2}, 2\right]$ and $\ell_{0}$ is large, observe that the support of (2.5) is disjoint from each of the bad hyperplanes

$$
\left\{\xi \in \mathbb{R}^{n}: \alpha_{1} \xi_{1}+\cdots+\alpha_{n-1} \xi_{n-1}+\xi_{n}=0\right\} \quad\left(\alpha \in\{0,1\}^{n-1}\right) .
$$

Thus, $\mu_{n}$ is smooth on the support of (2.5) (see Lemma 5.1) and therefore the desired bounds for the multilinear form with symbol (2.5) follow from (2.1).

Case 3 (The rough $\psi-\varphi$ case). It remains to consider $\psi$ - $\varphi$-type pieces where $\psi$ falls onto one of $\xi_{1}, \ldots, \xi_{n-1}$. By symmetry, we may restrict our attention to the symbol

$$
\sum_{k \in \mathbb{Z}} \mu_{n}(\xi) \psi_{k}\left(\xi_{1}\right) \varphi_{k}\left(\xi_{2}\right) \cdots \varphi_{k}\left(\xi_{n}\right)
$$

where $\varphi$ is supported in a small neighborhood of the origin. The support of this symbol is contained in a narrow cone around the $\xi_{1}$-axis and it has significant intersection with the singularities of $\mu_{n}$. In this case the idea is to reduce to a symbol that vanishes on the $\xi_{1}$-axis and then consider an appropriate lacunary decomposition with respect to that axis, where each of the pieces is a $\psi$ - $\psi$-type symbol which can be handled as in Case 1. This argument is detailed in $\$ 2.2$ We note that by inspection of the multiplier a simpler argument is available if $n=2$.

2.1. The $\psi-\psi$ case. In this section we bound the multilinear form corresponding to a symbol of the form

$$
\sum_{k \in \mathbb{Z}} \mu_{n}(\xi) \psi_{k}^{j_{0}}\left(\xi_{j_{0}}\right) \psi_{k}^{j_{1}}\left(\xi_{j_{1}}\right) \prod_{j \neq j_{0}, j_{1}} \varphi_{k}^{j}\left(\xi_{j}\right)
$$

where $1 \leq j_{0}<j_{1} \leq n$ and the $\psi^{j}, \varphi^{j}$ are of $\psi$-and $\varphi$-type, respectively. 
Based on the symmetries of the function $\mu_{n}$, we need to distinguish two cases: $\left(j_{0}, j_{1}\right)=(1,2)$ and $\left(j_{0}, j_{1}\right)=(1, n)$ (they are different only if $\left.n>2\right)$. However, the arguments we use for these two cases are, mutatis mutandis, identical. Thus, we will restrict our attention here to the case $\left(j_{0}, j_{1}\right)=(1, n)$. To improve readability we also suppose that the various $\psi$ - and $\varphi$-type functions are identical. This is again no loss of generality. With these reductions in mind, we are now left with the symbol

$$
\sum_{k \in \mathbb{Z}} \mu_{n}(\xi) \psi_{k}\left(\xi_{1}\right) \varphi_{k}\left(\xi_{2}\right) \cdots \varphi_{k}\left(\xi_{n-1}\right) \psi_{k}\left(\xi_{n}\right),
$$

where $\psi$ is a smooth function supported in $[1 / 2,2] \cup[-2,-1 / 2]$ and $\varphi$ is a smooth function supported in $[-2,2]$. Recall that

$$
\mu_{n}(\xi)=\int_{[0,1]^{n-1}} \operatorname{sgn}\left(\alpha_{1} \xi_{1}+\cdots+\alpha_{n-1} \xi_{n-1}+\xi_{n}\right) d \alpha .
$$

Our proof follows the argument of Muscalu Mus14b. The argument simplifies because we do not need to make use of the non-compact Littlewood-Paley projections used in [Mus14b]. The key observation that we will use (due to [Mus14b]) is that

$$
\mu_{n}(\xi)=\int_{[0,1]^{n-2}} \mu_{2}\left(\xi_{1}, \alpha_{2} \xi_{2}+\cdots+\alpha_{n-1} \xi_{n-1}+\xi_{n}\right) d\left(\alpha_{2}, \ldots, \alpha_{n-1}\right) .
$$

This will allow us to make use of the fact that the Fourier coefficients of $\mu_{2}$, when restricted to a Whitney box, decay quadratically ( see Lemma 2.1 below). For $\mu_{n}$ we seem to get only linear decay (see Mus14a]). We introduce the variable

$$
\eta=\eta_{\alpha, \xi}=\alpha_{2} \xi_{2}+\cdots+\alpha_{n-1} \xi_{n-1}+\xi_{n}
$$

and rewrite the quantity $\mu(\xi)$ as

$$
\int_{[0,1]^{n-2}} \sum_{k \in \mathbb{Z}} \rho\left(2^{-k}\left(\xi_{1}, \eta\right)\right) \psi_{k}\left(\xi_{1}\right) \varphi_{k}\left(\xi_{2}\right) \cdots \varphi_{k}\left(\xi_{n-1}\right) \psi_{k}\left(\xi_{n}\right) d\left(\alpha_{2}, \ldots, \alpha_{n-1}\right),
$$

where we have set

$$
\rho\left(\xi_{1}, \eta\right)=\mu_{2}\left(\xi_{1}, \eta\right) \widetilde{\psi}\left(\xi_{1}\right) \widetilde{\varphi}(\eta) .
$$

By the Fourier inversion formula we have

$$
\rho\left(\xi_{1}, \eta\right)=\int e^{-2 \pi i\left(\xi_{1} u+\eta v\right)} \check{\rho}(u, v) d(u, v) .
$$

At this point we record the following estimate for the function $\breve{\rho}$.

Lemma 2.1. For every $N \in \mathbb{N}, u, v \in \mathbb{R}$ we have

$$
|\breve{\rho}(u, v)| \lesssim_{N}(1+|v|)^{-2}\left((1+|v-u|)^{-N}+(1+|u|)^{-N}\right) .
$$

This is obtained by writing $\breve{\rho}(u, v)=\int e^{2 \pi i\left(\xi_{1} u+\eta v\right)} \mu_{2}\left(\xi_{1}, \eta\right) \widetilde{\psi}\left(\xi_{1}\right) \widetilde{\varphi}(\eta) d\left(\xi_{1}, \eta\right)$ and first integrating by parts twice with respect to $\eta$ and then $N$ times with respect to $\xi_{1}$. For the details we refer to Mus14a, Lemma 2.4]. Alternatively, the reader may look ahead at the details of our proof of (2.19) below, which is a slight variant of this estimate.

Note that the right hand side of (2.9) is integrable in $u, v \in \mathbb{R}$. Let us for the moment fix $\alpha_{2}, \ldots, \alpha_{n-1} \in[0,1], u, v \in \mathbb{R}$ and $k \in \mathbb{Z}$. After expanding $\rho\left(\xi_{1}, \eta\right)$ 
according to (2.8), the corresponding contribution to our multilinear form (2.4) becomes

$$
\begin{aligned}
& \int_{\left(\mathbb{R}^{n}\right)^{n}}\left(\widehat{F_{1}}\left(\tau_{1}\right) \psi_{k}\left(\tau_{11}\right) e^{-2 \pi i 2^{-k} \tau_{11} u}\right) \prod_{j=2}^{n-1}\left(\widehat{F_{j}}\left(\tau_{j}\right) \varphi_{k}\left(\tau_{j j}\right) e^{-2 \pi i 2^{-k} \alpha_{j j} \tau_{j j} v}\right) \\
& \quad \times\left(\widehat{F_{n}}\left(\tau_{n}\right) \psi_{k}\left(\tau_{n n}\right) e^{-2 \pi i 2^{-k} \tau_{n n} v}\right) \widehat{F_{0}}\left(-\left(\tau_{1}+\cdots+\tau_{n}\right)\right) d\left(\tau_{1}, \ldots, \tau_{n}\right),
\end{aligned}
$$

Expanding the Fourier transform $\widehat{F_{0}}$, the previous display becomes

$$
\begin{aligned}
\int_{\mathbb{R}^{n}} & \left(\int_{\mathbb{R}^{n}} \widehat{F_{1}}\left(\tau_{1}\right) \psi_{k}\left(\tau_{11}\right) e^{-2 \pi i\left(2^{-k} \tau_{11} u\right)} e^{2 \pi i x \cdot \tau_{1}} d \tau_{1}\right) \prod_{j=2}^{n-1} \\
& \times\left(\int_{\mathbb{R}^{n}} \widehat{F_{j}}\left(\tau_{j}\right) \varphi_{k}\left(\tau_{j j}\right) e^{-2 \pi i 2^{-k} \alpha_{j j} \tau_{j j} v} e^{2 \pi i x \cdot \tau_{j}} d \tau_{j}\right) \\
& \times\left(\int_{\mathbb{R}^{n}} \widehat{F_{n}}\left(\tau_{n}\right) \psi_{k}\left(\tau_{n n}\right) e^{-2 \pi i 2^{-k} \tau_{n n} v} e^{2 \pi i x \cdot \tau_{n}} d \tau_{n}\right) F_{0}(x) d x
\end{aligned}
$$

which by the Fourier inversion formula is equal to

$$
\int_{\mathbb{R}^{n}} F_{0}(x)\left(F_{1} *_{1}{\widetilde{\psi_{k}}}^{(u)}\right)(x) \prod_{j=2}^{n-1}\left(F_{j} *_{j}{\widetilde{\varphi_{k}}}^{\left(\alpha_{j} v\right)}\right)(x)\left(F_{n} *_{n}{\widetilde{\psi_{k}}}^{(v)}\right)(x) d x .
$$

Here $*_{j}$ denotes convolution in the $j$ th variable and $f^{(u)}(x)=f(x+u)$ denotes translation. Summing in $k \in \mathbb{Z}$ we obtain the multilinear form

$$
\int_{\mathbb{R}^{n}} F_{0}(x) \sum_{k \in \mathbb{Z}}\left(F_{1} *_{1}{\widetilde{\psi_{k}}}^{(u)}\right)(x) \prod_{j=2}^{n-1}\left(F_{j} *_{j}{\widetilde{\varphi_{k}}}^{\left(\alpha_{j} v\right)}\right)(x)\left(F_{n} *_{n}{\widetilde{\psi_{k}}}^{(v)}\right)(x) d x .
$$

Using the triangle inequality and the Cauchy-Schwarz inequality we estimate the absolute value of (2.11) by

$$
\begin{aligned}
\int_{\mathbb{R}^{n}}\left|F_{0}(x)\right|\left(\sum_{k \in \mathbb{Z}} \mid\right. & \left.\left|\left(F_{1} *_{1}{\widetilde{\psi_{k}}}^{(u)}\right)(x)\right|^{2}\right)^{1 / 2} \prod_{j=2}^{n-1} \sup _{k \in \mathbb{Z}}\left|\left(F_{j} *_{j} \widetilde{\varphi}_{k}^{\left(\alpha_{j} v\right)}\right)(x)\right| \\
& \times\left(\sum_{k \in \mathbb{Z}}\left|\left(F_{n} *_{n}{\widetilde{\psi_{k}}}^{(v)}\right)(x)\right|^{2}\right)^{1 / 2} d x
\end{aligned}
$$

At this point we need to make use of the following well-known bounds for shifted maximal functions and shifted square-functions.

Lemma 2.2. Let $u \in \mathbb{R}$, let $\varphi$ be a smooth bump function, and let $\psi$ be a $\psi$-type function. Then we have

$$
\left\|\sup _{k \in \mathbb{Z}}\left|f *{\widetilde{\varphi_{k}}}^{(u)}\right|\right\|_{p} \lesssim \log (2+|u|)\|f\|_{p}
$$

and

$$
\left\|\left(\sum_{k \in \mathbb{Z}}\left|f * \widetilde{\psi}_{k}^{(u)}\right|^{2}\right)^{1 / 2}\right\|_{p} \lesssim \log (2+|u|)\|f\|_{p}
$$

for every $p \in(1, \infty)$. 
For the proof of (2.13) we refer to [Mus14a, Theorem 4.1] (it is also contained in [Ste93]) and for the proof of (2.14) see [Mus14a, Theorem 5.1]. Using Hölder's inequality, (2.13), (2.14), and Fubini's theorem, we see that (2.12) is bounded by a constant times

$$
\prod_{j=0}^{n}\left\|F_{j}\right\|_{p_{j}}
$$

for all tuples $\left(p_{0}, \ldots, p_{n}\right)$ satisfying $\sum_{j=0}^{n} p_{j}^{-1}=1, p_{j} \in(1, \infty)\left(p_{j}=\infty\right.$ is also allowed unless $j \in\{1, n\})$ and the constant takes the form

$$
C \log (2+|u|) \log (2+|v|)
$$

where $C$ is an absolute constant. Integrating over $u, v \in \mathbb{R}, \alpha_{2}, \ldots, \alpha_{n-1} \in[0,1]$ and making use of Lemma 2.1 we obtain the desired bounds for our multilinear form.

2.2. The rough $\psi-\varphi$ case. In this section we deal with the symbol

$$
\sum_{k \in \mathbb{Z}} \mu_{n}(\xi) \psi_{k}\left(\xi_{1}\right) \varphi_{k}\left(\xi_{2}\right) \cdots \varphi_{k}\left(\xi_{n}\right)
$$

The difficulty is that the support of this function intersects some of the hyperplanes on which $\mu_{n}$ fails to be differentiable. The first step is again to use

$$
\mu_{n}(\xi)=\int_{[0,1]^{n-2}} \mu_{2}\left(\xi_{1}, \alpha_{2} \xi_{2}+\cdots+\alpha_{n-1} \xi_{n-1}+\xi_{n}\right) d\left(\alpha_{2}, \ldots, \alpha_{n-1}\right) .
$$

We again consider the variable

$$
\eta=\eta_{\alpha, \xi}=\alpha_{2} \xi_{2}+\cdots+\alpha_{n-1} \xi_{n-1}+\xi_{n} .
$$

Fixing $\alpha_{2}, \ldots, \alpha_{n-1} \in[0,1]$, we now study the symbol

$$
\sum_{k \in \mathbb{Z}} \mu_{2}\left(\xi_{1}, \eta\right) \psi_{k}\left(\xi_{1}\right) \prod_{j=2}^{n} \varphi_{k}\left(\xi_{j}\right) .
$$

We split $\psi=\psi^{+}+\psi^{-}$such that $\psi^{+}$is supported on $\{\eta>0\}$ and $\psi^{-}$is supported on $\{\eta<0\}$. Note that $\psi^{+}$and $\psi^{-}$are smooth. We split the symbol accordingly into two pieces. By symmetry it suffices to consider the symbol

$$
\sum_{k \in \mathbb{Z}} \mu_{2}\left(\xi_{1}, \eta\right) \psi_{k}^{+}\left(\xi_{1}\right) \prod_{j=2}^{n} \varphi_{k}\left(\xi_{j}\right) .
$$

Using (2.1), we may subtract the smooth symbol $\sum_{k \in \mathbb{Z}} \psi_{k}^{+}\left(\xi_{1}\right) \prod_{j=2}^{n} \varphi_{k}\left(\xi_{j}\right)$ so that we are now concerned with

$$
\sum_{k \in \mathbb{Z}} \widetilde{\mu}_{2}\left(\xi_{1}, \eta\right) \psi_{k}^{+}\left(\xi_{1}\right) \prod_{j=2}^{n} \varphi_{k}\left(\xi_{j}\right),
$$

where we have set

$$
\widetilde{\mu}\left(\xi_{1}, \eta\right)=\mu_{2}\left(\xi_{1}, \eta\right)-1=-2 \int_{0}^{1} \mathbf{1}_{<0}\left(\xi_{1} \alpha+\eta\right) d \alpha .
$$

Now we perform another paraproduct decomposition. More precisely, we expand each of the $\varphi_{k}\left(\xi_{j}\right)$ into an appropriate sum of $\psi$-type functions and distinguish $n-1$ 
terms based on which of the summation indices is the largest (as in (2.2)). With this in mind it suffices to consider the symbol

$$
\sum_{\ell>\ell_{0}}\left(\sum_{k \in \mathbb{Z}} \widetilde{\mu}\left(\xi_{1}, \eta\right) \psi_{k}^{+}\left(\xi_{1}\right) \psi_{k-\ell}\left(\xi_{2}\right) \prod_{j=3}^{n} \varphi_{k-\ell}\left(\xi_{j}\right)\right) .
$$

In the following we will fix $\ell>\ell_{0}$ and bound the multilinear form corresponding to the inner sum with summable decay in $\ell$. Shifting the index $k$ and using homogeneity of $\mu$ we can write the inner sum as

$$
\sum_{k \in \mathbb{Z}} \widetilde{\mu}\left(2^{-k}\left(\xi_{1}, \eta\right)\right) \psi_{k+\ell}^{+}\left(\xi_{1}\right) \psi_{k}\left(\xi_{2}\right) \prod_{j=3}^{n} \varphi_{k}\left(\xi_{j}\right) .
$$

Observe that on the support of each summand we have

$$
|\eta| \leq\left|\xi_{2}\right|+\cdots+\left|\xi_{n-1}\right|+\left|\xi_{n}\right| \leq 2 n \cdot 2^{-k} .
$$

Choose a smooth function $\widetilde{\varphi}$ such that it equals 1 on the interval $[-2 n, 2 n]$ and is equal to 0 on a slightly larger interval. Similarly, we choose a smooth function $\widetilde{\psi}$ which is 1 on the interval $\left[\frac{1}{2}, 2\right]$ and equal to 0 on, say, $\left[\frac{1}{2}-\frac{1}{100}, 2+\frac{1}{100}\right]$. Then (2.16) is equal to

$$
\sum_{k \in \mathbb{Z}} \widetilde{\mu}\left(2^{-k}\left(\xi_{1}, \eta\right)\right) \widetilde{\psi}_{k+\ell}\left(\xi_{1}\right) \widetilde{\varphi}_{k}(\eta) \psi_{k+\ell}^{+}\left(\xi_{1}\right) \psi_{k}\left(\xi_{2}\right) \prod_{j=3}^{n} \varphi_{k}\left(\xi_{j}\right),
$$

which we rewrite as

$$
\sum_{k \in \mathbb{Z}} m_{\ell}\left(2^{-k-\ell} \xi_{1}, 2^{-k} \eta\right) \psi_{k+\ell}\left(\xi_{1}\right) \psi_{k}\left(\xi_{2}\right) \prod_{j=3}^{n} \varphi_{k}\left(\xi_{j}\right)
$$

where

$$
m_{\ell}\left(\xi_{1}, \eta\right)=\widetilde{\mu}\left(\xi_{1}, 2^{-\ell} \eta\right) \widetilde{\psi}\left(\xi_{1}\right) \widetilde{\varphi}(\eta) .
$$

Here we have used homogeneity of $\widetilde{\mu}$. By the Fourier inversion formula we have

$$
m_{\ell}\left(2^{-k-\ell} \xi_{1}, 2^{-k} \eta\right)=\int_{\mathbb{R}^{2}} e^{-2 \pi i\left(u 2^{-k-\ell} \xi_{1}+i v 2^{-k} \eta\right)} \widetilde{m}_{\ell}(u, v) d(u, v) .
$$

Suppose for a moment that we can prove the decay estimate

$$
\left|\widetilde{m}_{\ell}(u, v)\right| \lesssim_{N} 2^{-\ell}(1+|v|)^{-2}(1+|u|)^{-N}
$$

for all $\ell>\ell_{0}$. Then we insert (2.18) into (2.17) and fix $u, v \in \mathbb{R}$. The corresponding contribution to the multilinear form has the symbol

$$
\begin{aligned}
\sum_{k \in \mathbb{Z}}( & \left.\psi_{k+\ell}\left(\xi_{1}\right) e^{-2 \pi i u 2^{-(k+\ell)} \xi_{1}}\right)\left(\psi_{k}\left(\xi_{2}\right) e^{-i v 2^{-k} \alpha_{2} \xi_{2}}\right) \\
& \times\left(\prod_{j=3}^{n-1} \varphi_{k}\left(\xi_{j}\right) e^{-i v 2^{-k} \alpha_{n-1} \xi_{n-1}}\right)\left(\varphi_{k}\left(\xi_{n}\right) e^{-i v 2^{-k} \xi_{n}}\right) .
\end{aligned}
$$

Repeating the same argument as in the $\psi-\psi$ case (more specifically, repeating the steps indicated from (2.10) to (2.15) ), we obtain a bound that depends only logarithmically on $u, v$ and does not depend on $\ell$ (it does not depend on $\ell$, because one can shift each index $k$ appearing in (2.12) separately). The estimate (2.19) then 
allows us to sum these bounds in $\ell$ and integrate in $u, v \in \mathbb{R}$. It now only remains to verify (2.19). We write

$$
\widetilde{m_{\ell}}(u, v)=\int_{\mathbb{R}^{2}} e^{2 \pi i\left(u \xi_{1}+v \eta\right)} \widetilde{\mu}\left(\xi_{1}, 2^{-\ell} \eta\right) \widetilde{\psi}\left(\xi_{1}\right) \widetilde{\varphi}(\eta) d\left(\xi_{1}, \eta\right) .
$$

Note that on the support of the integrand we have $\xi_{1}>0, \eta<0$ and $\left|2^{-\ell} \eta / \xi_{1}\right|<1$ and for such $\left(\xi_{1}, \eta\right)$ we have

$$
\widetilde{\mu}\left(\xi_{1}, 2^{-\ell} \eta\right)=-2 \int_{0}^{1} \mathbf{1}_{<0}\left(\alpha+2^{-\ell} \eta / \xi_{1}\right) d \alpha=2^{-\ell+1} \frac{\eta}{\xi_{1}} .
$$

On the other hand, if $\eta>0$, then $\widetilde{\mu}\left(\xi_{1}, 2^{-\ell} \eta\right)=0$. Therefore (2.20) is equal to

$$
2^{-\ell+1}\left(\int_{-\infty}^{0} e^{2 \pi i v \eta} \widetilde{\varphi}(\eta) \eta d \eta\right)\left(\int_{\mathbb{R}} e^{2 \pi i u \xi_{1}} \widetilde{\psi}\left(\xi_{1}\right) \xi_{1}^{-1} d \xi_{1}\right) .
$$

Integrating by parts $N$ times in $\xi_{1}$ yields the decaying factor $(1+|u|)^{-N}$. In the $\eta$ variable we may only integrate by parts twice, which yields

$$
\int_{-\infty}^{0} e^{2 \pi i v \eta} \widetilde{\varphi}(\eta) \eta d \eta=\frac{1}{(2 \pi i v)^{2}}\left(-1+\int_{-\infty}^{0} e^{2 \pi i v \eta}\left(\widetilde{\varphi}^{\prime \prime}(\eta) \eta+2 \widetilde{\varphi}^{\prime}(\eta)\right) d \eta\right) .
$$

Thus we have verified (2.19).

\section{An estimate in $\mathbb{R}^{3}$}

In this section we indicate how to prove (1.10) in the case $n=3$. The argument closely follows Kovač's proof for boundedness of the twisted paraproduct [Kov12] and here we only provide the necessary changes in his argument. By a standard cone decomposition (see [Thi06]) the symbol can be written as a certain superposition of symbols of the form

$$
\begin{aligned}
& m_{1}\left(\xi_{1}, \xi_{2}, \xi_{3}\right)=\sum_{k \in \mathbb{Z}} c_{k} \psi_{k}\left(\xi_{1}\right) \varphi_{k}\left(\xi_{2}\right) \varphi_{k}\left(\xi_{3}\right), \\
& m_{2}\left(\xi_{1}, \xi_{2}, \xi_{3}\right)=\sum_{k \in \mathbb{Z}} c_{k} \varphi_{k}\left(\xi_{1}\right) \psi_{k}\left(\xi_{2}\right) \varphi_{k}\left(\xi_{3}\right), \\
& m_{3}\left(\xi_{1}, \xi_{2}, \xi_{3}\right)=\sum_{k \in \mathbb{Z}} c_{k} \varphi_{k}\left(\xi_{1}\right) \varphi_{k}\left(\xi_{2}\right) \psi_{k}\left(\xi_{3}\right),
\end{aligned}
$$

where the coefficients $c_{k}$ satisfy $\left|c_{k}\right| \leq 1, \varphi$ is a $\varphi$-type function, and $\psi$ a $\psi$-type function as in $\oiint_{2}$. Without loss of generality we will assume that $c_{k}=1$ for all $k$ (see [Kov12, §1]). Each of $m_{1}, m_{2}, m_{3}$ is treated in the same way. We focus on $m_{3}$. The corresponding multilinear form can be written as

$$
\Lambda_{m_{3}}\left(F_{0}, F_{1}, F_{2}, F_{3}\right)=\left\langle T_{c}\left(F_{1}, F_{2}, F_{3}\right), F_{4}\right\rangle,
$$

where

$$
T_{c}\left(F_{1}, F_{2}, F_{3}\right)=\sum_{k \in \mathbb{Z}}\left(F_{1} *_{1} \widetilde{\varphi_{k}}\right)\left(F_{2} *_{2} \widetilde{\varphi_{k}}\right)\left(F_{3} *_{3} \widetilde{\psi_{k}}\right),
$$

where $*_{j}$ denotes convolution in the $j$ th variable. We now pass to a dyadic model operator for $T_{c}$. By $\mathcal{D}$ we denote the collection of dyadic intervals in $\mathbb{R}$. We define the Haar function $\psi_{I}$ adapted to $I \in \mathcal{D}$ by

$$
\psi_{I}=|I|^{-1 / 2}\left(\mathbf{1}_{I_{\mathrm{L}}}-\mathbf{1}_{I_{\mathrm{R}}}\right),
$$


where $I_{\mathrm{L}}, I_{\mathrm{R}}$ denote the left and right dyadic subintervals of $I$. Also write $\varphi_{I}=$ $|I|^{-1 / 2} \mathbf{1}_{I}$. Denote dyadic martingale averages and differences by

$$
\mathbb{E}_{k} f=\sum_{I \in \mathcal{D},|I|=2^{-k}}\left\langle f, \varphi_{I}\right\rangle \varphi_{I}, \quad \Delta_{k} f=\sum_{I \in \mathcal{D},|I|=2^{-k}}\left\langle f, \psi_{I}\right\rangle \psi_{I}=\mathbb{E}_{k+1} f-\mathbb{E}_{k} f .
$$

Here $\langle f, g\rangle$ denotes $\int f g$. If $F$ is a function on $\mathbb{R}^{3}$ and $j \in\{1,2,3\}$, we write $\mathbb{E}_{k}^{j} F$, $\Delta_{k}^{j} F$ to denote application of $\mathbb{E}_{k}$ or $\Delta_{k}$ in the $j$ th variable, respectively. We define a trilinear operator by

$$
T_{d}\left(F_{1}, F_{2}, F_{3}\right)=\sum_{k \in \mathbb{Z}}\left(\mathbb{E}_{k}^{1} F_{1}\right)\left(\mathbb{E}_{k}^{2} F_{2}\right)\left(\Delta_{k}^{3} F_{3}\right) .
$$

Let us suppose for a moment that we can prove

$$
\left\|T_{d}\left(F_{1}, F_{2}, F_{3}\right)\right\|_{p_{0}^{\prime}} \lesssim \prod_{j=1}^{3}\left\|F_{j}\right\|_{p_{j}}
$$

for $\left(p_{0}, p_{1}, p_{2}, p_{3}\right)$ satisfying $\sum_{j=0}^{3} p_{j}=1$ and $p_{0}, p_{1}, p_{2} \in(4, \infty), p_{3} \in[2, \infty)$ and $p_{0}^{\prime}$ the Hölder dual exponent of $p_{0}$. We can then use a square-function estimate due to Jones, Seeger, and Wright JSW08 comparing martingale averages with Littlewood-Paley projections to deduce the inequality

$$
\left\|T_{c}\left(F_{1}, F_{2}, F_{3}\right)\right\|_{p_{0}^{\prime}} \lesssim \prod_{j=1}^{3}\left\|F_{j}\right\|_{p_{j}}
$$

for $\left(p_{0}, p_{1}, p_{2}, p_{3}\right)$ satisfying $\sum_{j=0}^{3} p_{j}=1$ and $p_{0}, p_{1}, p_{2} \in(4, \infty), p_{3} \in[2, \infty)$. This argument is detailed in [Kov12, §6] in the case of the (two-dimensional) twisted paraproduct. Up to obvious modifications this argument also applies to our case, so we omit the details.

Using multilinear interpolation and the fiber-wise Calderón-Zygmund decomposition due to Bernicot Ber12] we can extend the range of exponents in this estimate to obtain (3.2) for $p_{0}, p_{1}, p_{2}, p_{3} \in(1, \infty)$ satisfying $\sum_{j=0}^{3} p_{j}^{-1}=1, p_{0}^{-1}<2^{-2}$, $p_{3}^{-1}>2^{-2}, p_{1}^{-1}+p_{2}^{-1}>2^{-2}, p_{2}^{-1}+p_{3}^{-1}>2^{-1}$, and $p_{1}^{-1}+p_{3}^{-1}>2^{-1}$. This follows in the same way as in Bernicot's original argument up to obvious modifications, so we leave it out. By duality, this implies bounds for the form $\Lambda_{m_{3}}$. Arguing by symmetry, we also obtain corresponding bounds for $\Lambda_{m_{1}}$ and $\Lambda_{m_{2}}$. Altogether, we obtain bounds for the form $\Lambda_{m}$ associated with the original symbol $m$ that are valid for exponent tuples $\left(p_{0}, p_{1}, p_{2}, p_{3}\right)$ that lie in the intersection of the three regions of exponents stemming from $\Lambda_{m_{1}}, \Lambda_{m_{2}}, \Lambda_{m_{3}}$, respectively. This region consists of all $\left(p_{0}, p_{1}, p_{2}, p_{3}\right) \in(4, \infty) \times(1,4)^{3}$ so that $\sum_{j=0}^{3} p_{j}^{-1}=1$.

It now only remains to address the validity of the estimate (3.1). This estimate follows from the techniques in Kov12. The details can be found in Dur14] (see $\S 4.4$ there). For the reader's convenience we also sketch the estimate here in the special case that $p_{3}=2$. It can be proven in the local $L^{2}$ range $p_{3} \in(2, \infty)$ by considering an appropriate local form as in [Kov12, §3]. For this purpose it is again convenient to consider a multilinear form. We write

$$
\Lambda_{d}\left(F_{0}, F_{1}, F_{2}, F_{3}\right)=\left\langle T_{d}\left(F_{1}, F_{2}, F_{3}\right), F_{0}\right\rangle .
$$

Without loss of generality we may assume that the functions $F_{j}$ are non-negative. This is by splitting both the real and imaginary parts into positive and negative 
parts each. Expanding the martingale averages and differences and using Fubini's theorem, we see that $\Lambda_{d}\left(F_{0}, F_{1}, F_{2}, F_{3}\right)$ is equal to

$$
\begin{gathered}
\sum_{Q} \int_{\mathbb{R}^{4}}\left(\int_{\mathbb{R}} F_{0}(x, y, z) F_{1}\left(x^{\prime}, y, z\right) F_{2}\left(x, y^{\prime}, z\right) \psi_{I_{3}}(z) d z\right)\left(\int_{\mathbb{R}} F_{3}\left(x, y, z^{\prime}\right) \psi_{I_{3}}\left(z^{\prime}\right) d z^{\prime}\right) \\
\times \varphi_{I_{1}}(x) \varphi_{I_{1}}\left(x^{\prime}\right) \varphi_{I_{2}}(y) \varphi_{I_{2}}\left(y^{\prime}\right) d\left(x, y, x^{\prime}, y^{\prime}\right)
\end{gathered}
$$

where the sum is over all dyadic cubes $Q=I_{1} \times I_{2} \times I_{3} \subset \mathbb{R}^{3}$. By the CauchySchwarz inequality this is no greater than

$$
\begin{aligned}
\left(\sum _ { Q } \int _ { \mathbb { R } ^ { 4 } } \left(\int_{\mathbb{R}} F_{0}(x, y, z) F_{1}(\right.\right. & \left.\left.x^{\prime}, y, z\right) F_{2}\left(x, y^{\prime}, z\right) \psi_{I_{3}}(z) d z\right)^{2} \\
& \left.\times \varphi_{I_{1}}(x) \varphi_{I_{1}}\left(x^{\prime}\right) \varphi_{I_{2}}(y) \varphi_{I_{2}}\left(y^{\prime}\right) d\left(x, y, x^{\prime}, y^{\prime}\right)\right)^{1 / 2}
\end{aligned}
$$

times

$$
\begin{aligned}
\left(\sum_{Q} \int_{\mathbb{R}^{4}}\left(\int_{\mathbb{R}} F_{3}\left(x, y, z^{\prime}\right) \psi_{I_{3}}\left(z^{\prime}\right) d z^{\prime}\right)^{2} \varphi_{I_{1}}(x) \varphi_{I_{1}}\right. & \left(x^{\prime}\right) \varphi_{I_{2}}(y) \\
& \left.\times \varphi_{I_{2}}\left(y^{\prime}\right) d\left(x, y, x^{\prime}, y^{\prime}\right)\right)^{1 / 2}
\end{aligned}
$$

We will treat these two factors separately. Integrating in $x^{\prime}, y^{\prime}$, 3.4) becomes

$$
\left(\sum_{k \in \mathbb{Z}} \sum_{\left|I_{3}\right|=2^{-k}} \int_{\mathbb{R}^{4}} F_{3}(x, y, z) F_{3}\left(x, y, z^{\prime}\right) \psi_{I_{3}}(z) \psi_{I_{3}}\left(z^{\prime}\right) d\left(x, y, z, z^{\prime}\right)\right)^{1 / 2},
$$

which equals $\left\|F_{3}\right\|_{2}$. On the other hand, by Theorem 6 from Kov12 applied to the 6-tuple $\left(F_{0}, F_{0}, F_{1}, F_{1}, F_{2}, F_{2}\right)$ we obtain that the square of (3.3) is bounded by

$$
\lesssim\left\|F_{0}\right\|_{p_{0}}^{2}\left\|F_{1}\right\|_{p_{1}}^{2}\left\|F_{2}\right\|_{p_{2}}^{2}
$$

where $\frac{1}{p_{0}}+\frac{1}{p_{1}}+\frac{1}{p_{2}}=\frac{1}{2}$ and $p_{0}, p_{1}, p_{2} \in(4, \infty)$.

\section{Endpoint COUntereXAMPLE}

Let $n \geq 2$ and $j_{0} \in\{1, \ldots, n\}$. Let $p_{j_{0}}=\infty$ and choose the other $\left(p_{j}\right)_{j}$ from $[1, \infty]$ such that $\sum_{j=0}^{n} p_{j}^{-1}=1$. We claim that the estimate (1.2) fails. Let $N \gg 1$ and set

$$
\begin{gathered}
F_{0}(x)=\mathbf{1}_{(0,1)}\left(x_{j_{0}}\right) \prod_{j \neq j_{0}} \mathbf{1}_{(0, N)}\left(x_{j}\right), \quad F_{j_{0}}(x)=\mathbf{1}_{>0}\left(x_{j_{0}}\right), \\
F_{j}(x)=\mathbf{1}_{(0,1)}\left(x_{j_{0}}\right) \prod_{\ell \neq j_{0}} \mathbf{1}_{(-N, N)}\left(x_{\ell}\right) \quad \text { for } j \neq 0, j_{0} .
\end{gathered}
$$

Clearly, $\left\|F_{j_{0}}\right\|_{\infty}=1$ and $\left\|F_{j}\right\|_{p_{j}} \approx N^{(n-1) / p_{j}}$ for $j \neq j_{0}$. Thus, the right hand side of (1.2) equals $N^{n-1}$. By a straightforward computation the left hand side of (1.2) is comparable to $N^{n-1} \log (N)$. Letting $N \rightarrow \infty$ implies the claim. 


\section{Some comments on the Multiplier}

In this section we are concerned with the function $\mu_{n}$ defined in (1.8). This function is the symbol of our multilinear form (1.1) and also conincides with the symbol of the Calderón commutator (1.5). Apart from the quadratic Fourier decay of $\mu_{2}$ (see Lemma 2.1) the only additional information which we need in $\$ 2$ is the following.

Lemma 5.1. The function $\mu_{n}$ is smooth on the complement of the hyperplanes

$$
\left\{\xi \in \mathbb{R}^{n}: \alpha_{1} \xi_{1}+\cdots+\alpha_{n-1} \xi_{n-1}+\xi_{n}=0\right\}
$$

where $\alpha \in\{0,1\}^{n-1}$.

This is a routine verification. For the convenience of the reader we include some details here. First, note the following explicit formula:

$$
\mu_{n}(\xi)=\frac{c_{n}}{\xi_{1} \ldots \xi_{n-1}} \sum_{\alpha \in\{0,1\}^{n-1}}(-1)^{|\alpha|} g_{n}\left(\xi_{n}+\sum_{j=1}^{n-1} \alpha_{j} \xi_{j}\right),
$$

where $g_{n}(t)=|t| t^{n-2}, c_{n}=\frac{(-1)^{n+1}}{(n-1) !}$, and $\xi=\left(\xi_{1}, \ldots, \xi_{n}\right), \alpha=\left(\alpha_{1}, \ldots, \alpha_{n-1}\right)$. This formula holds if $\xi_{1}, \ldots, \xi_{n-1} \neq 0$. If $k \geq 1$ of the numbers $\xi_{1}, \ldots, \xi_{n-1}$ are equal to zero, then

$$
\mu_{n}(\xi)=\mu_{n-k}\left(\xi_{*}\right)
$$

where $\xi_{*}$ is the vector in $\mathbb{R}^{n-k}$ obtained by removing the zero coordinates among $\xi_{1}, \ldots, \xi_{n-1}$. This is proven by induction on $n$. Let us denote the union of the hyperplanes (5.1) by B. Then $\mathbb{R}^{n} \backslash \mathbf{B}$ consists of a finite number of connected components each of which is a convex polytope that we call a sector. Each sector corresponds to a map $\sigma:\{0,1\}^{n-1} \rightarrow\{-1,1\}$ where $\sigma(\alpha)$ prescribes the sign of $\xi_{n}+\sum_{j=1}^{n-1} \alpha_{j} \xi_{j}$. On the closure of each such sector $\mu_{n}$ is given by a homogeneous rational function with no singularities in the closure of the sector. This in particular implies that $\mu_{n}$ is smooth on $\mathbb{R}^{n} \backslash \mathbf{B}$.

Remark. It is natural to ask how many of the $2^{2^{n-1}}$ maps $\sigma$ correspond to (nonempty) sectors. To this end we observe that there is a bijection between sectors and linear boolean threshold functions on the $(n-1)$-hypercube. A threshold function on the $n$-cube $\{-1,1\}^{n}$ is a map $f:\{-1,1\}^{n} \rightarrow\{-1,1\}$ that takes the form $f(x)=\operatorname{sgn}(a+\langle x, b\rangle)$ for some $a \in \mathbb{R}, b \in \mathbb{R}^{n}$. Threshold functions are of practical relevance in pattern recognition and have been studied extensively in the computer science literature Mur71. In particular, it has been shown that $2^{n^{2}+o\left(n^{2}\right)} \leq \#_{n} \leq 2^{n^{2}}$ (see [Mur71], Zue89]), where $\#_{n}$ is the number of threshold functions on $\{-1,1\}^{n}$. In view of the separating hyperplanes theorem, the number $\#_{n}$ also has a simple geometric interpretation: it equals the number of subsets $A$ of vertices of the $n$-cube such that the convex hull of $A$ is disjoint from the convex hull of the vertices not in $A$.

\section{ACKNowledgments}

We thank Vjeko Kovač for helpful comments on a preliminary draft and Lenka Slavíková for pointing out an inaccuracy in a previous version of this paper. 


\section{REFERENCES}

[Ber12] Frédéric Bernicot, Fiber-wise Calderón-Zygmund decomposition and application to a bi-dimensional paraproduct, Illinois J. Math. 56 (2012), no. 2, 415-422. MR3161332

[CMM82] R. R. Coifman, A. McIntosh, and Y. Meyer, L'intégrale de Cauchy définit un opérateur borné sur $L^{2}$ pour les courbes lipschitziennes (French), Ann. of Math. (2) 116 (1982), no. 2, 361-387, DOI 10.2307/2007065. MR672839

[DT10] Ciprian Demeter and Christoph Thiele, On the two-dimensional bilinear Hilbert transform, Amer. J. Math. 132 (2010), no. 1, 201-256, DOI 10.1353/ajm.0.0101. MR2597511

[Dur14] P. Durcik. $L^{p}$ estimates for entangled multilinear forms. Master Thesis, University of Bonn, 2014.

[DKT16] Polona Durcik, Vjekoslav Kovač, and Christoph Thiele, Power-type cancellation for the simplex Hilbert transform, J. Anal. Math. 139 (2019), no. 1, 67-82, DOI 10.1007/s11854-019-0052-4. MR 4041095

[DT18] P. Durcik, C. Thiele, Singular Brascamp-Lieb inequalities, arXiv: 1809.08688. Preprint, 2018.

[GT02] Loukas Grafakos and Rodolfo H. Torres, Multilinear Calderón-Zygmund theory, Adv. Math. 165 (2002), no. 1, 124-164, DOI 10.1006/aima.2001.2028. MR.1880324

[JSW08] Roger L. Jones, Andreas Seeger, and James Wright, Strong variational and jump inequalities in harmonic analysis, Trans. Amer. Math. Soc. 360 (2008), no. 12, 6711-6742, DOI 10.1090/S0002-9947-08-04538-8. MR2434308

[Kov12] Vjekoslav Kovač, Boundedness of the twisted paraproduct, Rev. Mat. Iberoam. 28 (2012), no. 4, 1143-1164, DOI 10.4171/RMI/707. MR2990138

[KTZ15] Vjekoslav Kovač, Christoph Thiele, and Pavel Zorin-Kranich, Dyadic triangular Hilbert transform of two general functions and one not too general function, Forum Math. Sigma 3 (2015), e25, 27, DOI 10.1017/fms.2015.25. MR3482272

[Mur71] Saburo Muroga, Threshold logic and its applications, Wiley-Interscience [John Wiley \& Sons], New York-London-Sydney, 1971. MR0439441

[Mus14a] Camil Muscalu, Calderón commutators and the Cauchy integral on Lipschitz curves revisited: I. First commutator and generalizations, Rev. Mat. Iberoam. 30 (2014), no. 2, 727-750, DOI 10.4171/RMI/798. MR3231215

[Mus14b] Camil Muscalu, Calderón commutators and the Cauchy integral on Lipschitz curves revisited II. The Cauchy integral and its generalizations, Rev. Mat. Iberoam. 30 (2014), no. 3, 1089-1122, DOI 10.4171/RMI/808. MR.3255002

[Pal12] Eyvindur Ari Palsson, $L^{p}$ estimates for a singular integral operator motivated by Calderón's second commutator, J. Funct. Anal. 262 (2012), no. 4, 1645-1678, DOI 10.1016/j.jfa.2011.11.014. MR2873854

[Ste93] Elias M. Stein, Harmonic analysis: real-variable methods, orthogonality, and oscillatory integrals, Princeton Mathematical Series, vol. 43, Princeton University Press, Princeton, NJ, 1993. With the assistance of Timothy S. Murphy; Monographs in Harmonic Analysis, III. MR:1232192

[Tao16] Terence Tao, Cancellation for the multilinear Hilbert transform, Collect. Math. 67 (2016), no. 2, 191-206, DOI 10.1007/s13348-015-0162-y. MR3484017

[Thi06] Christoph Thiele, Wave packet analysis, CBMS Regional Conference Series in Mathematics, vol. 105, Published for the Conference Board of the Mathematical Sciences, Washington, DC; by the American Mathematical Society, Providence, RI, 2006. MR2199086

[LT97] Michael Lacey and Christoph Thiele, $L^{p}$ estimates on the bilinear Hilbert transform for $2<p<\infty$, Ann. of Math. (2) 146 (1997), no. 3, 693-724, DOI 10.2307/2952458. MR 1491450

[LT99] Michael Lacey and Christoph Thiele, On Calderón's conjecture, Ann. of Math. (2) 149 (1999), no. 2, 475-496, DOI 10.2307/120971. MR 1689336

[Zor17] Pavel Zorin-Kranich, Cancellation for the simplex Hilbert transform, Math. Res. Lett. 24 (2017), no. 2, 581-592, DOI 10.4310/MRL.2017.v24.n2.a16. MR3685286

[Zue89] Yu. A. Zuev, Asymptotics of the logarithm of the number of Boolean threshold functions (Russian), Dokl. Akad. Nauk SSSR 306 (1989), no. 3, 528-530; English transl., Soviet Math. Dokl. 39 (1989), no. 3, 512-513. MR.1009467 
Department of Mathematics, California Institute of Technology, 1200 E California Boulevard, Pasadena, California 91125

Current address: Schmid College of Science and Technology, Chapman University, One University Drive, Orange, California 92866

Email address: durcik@chapman.edu

Department of Mathematics, University of Wisconsin-Madison, 480 Lincoln Drive, MADison, Wisconsin 53706

Current address: Department of Mathematical Sciences, University of Massachusetts Lowell, Lowell, Massachusetts 01854

Email address: joris_roos@uml.edu 